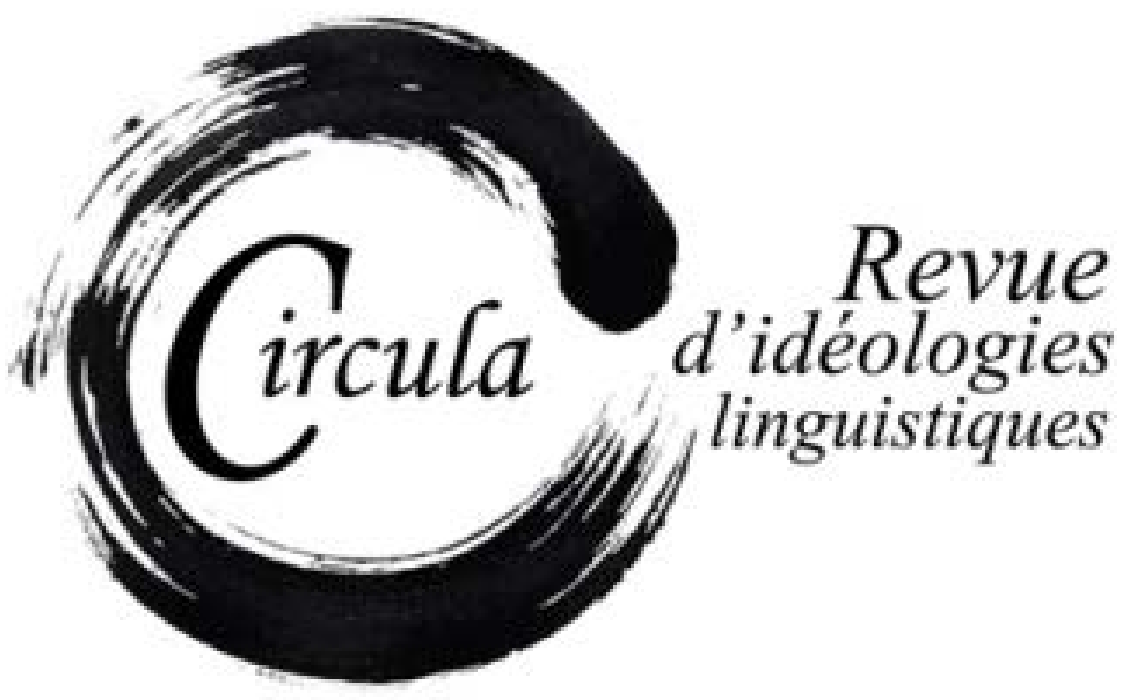

TITRE: ÉTUDIANTS ET APPRENANTS DE CATALAN EN CATALOGNE DU NORD : ÉCHO DU CONFLIT DIGLOSSIQUE ESPAGNOL

Auteur(s): Thierry Trefault, Université Paul-Valéry - MontPellier 3

Revue: CirCula, NUMÉRO 3

PAGES: $20-42$

ISSN: 2369-6761

Directeurs: Bruno MaUrer, Université PAul-ValÉRy - MontPellier 3

URI: HTTP://HDL.HANDLE.NET/11143/9703

DOI: $10.17118 / 11143 / 9703$ 


\section{Étudiants et apprenants de catalan en Catalogne du nord : écho du conflit diglossique espagnol}

Thierry Trefault, Université Paul-Valéry - Montpellier 3 thierry.trefault@montpellier.iufm.fr

Résumé : Dans le département des Pyrénées orientales, nombreux sont ceux qui apprennent le catalan, langue régionale aux côtés du français. Cet espace géographique plurilingue se rattache à l'entité plus vaste de la Catalogne, berceau de conflits diglossique virulents, au cœur de revendications d'autonomie qui secouent le climat politique actuel en Espagne. Cet article rend compte d'une enquête réalisée dans le cadre du projet « Représentations des langues et des identités en Méditerranée en contexte plurilingue » (EA 739 Dipralang). II s'agit de mettre en évidence les représentations du catalan et du français chez les apprenants de catalan, qu'ils soient locuteurs natifs, étudiants à l'université, dans les filières spécifiques ou comme option, ou encore qu'ils se destinent à l'enseigner dans les classes bilingues. Nous faisons l'hypothèse que ces représentations diffèrent en fonction de l'implication dans la diglossie français-catalan et qu'elles sont un écho au conflit linguistique propre à la Catalogne. Pour la vérifier, nous utilisons la méthode d'analyse combinée mise au point par Bruno Maurer.

Mots-clés : représentations des langues; conflit diglossique ; Catalogne nord

Abstract: In the Pyrénées-Orientales department, many people learn Catalan, the regional language alongside French. This multilingual geographical area is closely linked to the larger entity of Catalonia, the hotbed of vehement diglossic conflicts, at the centre of a struggle for autonomy that has been disrupting the current political climate in Spain. This article reports on a survey conducted within the project "Representations des langues et des identités en Méditerranée en contexte plurilingue" (EA 739 Dipralang). This project aims to highlight different representations of Catalan and French amongst learners of Catalan, whether they are native speakers, university students - either majoring in the language or taking it as an option -, or intending on teaching bilingual classes. We hypothesize that these representations differ depending on the students involvement in the French-Catalan diglossia, and that they echo a linguistic conflict specific to Catalonia. In order to verify this hypothesis, we will be using the combined analytical method developed by Bruno Maurer.

Keywords: representations of languages; diglossic conflict; North Catalonia 


\section{Introduction}

L'enquête sociolinguistique dont il va être question dans ces pages a lieu au moment où les Catalans de la partie espagnole organisent un référendum pour demander l'autonomie de leur province. De nombreuses manifestations ont précédé cet événement, auxquelles se sont joints des catalans français, solidaires de ces revendications. La culture et surtout la langue catalane tiennent une place de premier plan dans cette demande de reconnaissance, indice que le « conflit diglossique » est une réalité de chaque côté des Pyrénées.

Se pose-t-il cependant dans les mêmes termes ? À première vue non, car l'histoire a fait suffisamment diverger les deux régions, séparées depuis trois siècles et demi et immergées dans deux pays européens, qui suivent l'un et l'autre un développement trop différent pour qu'une comparaison puisse être envisagée. La Catalogne du nord est incluse dans le département français des Pyrénées orientales avec lequel elle se confond pratiquement. Quand on réside dans ce département sans en être originaire, on ressent très vite la singularité et l'identité de cette région, bien circonscrite par deux massifs montagneux, les collines des Corbières au nord et la chaîne de Pyrénées terminant sa course dans la Méditerranée, le tout dominé par les sommets enneigés du Canigou. Les emblèmes de la « catalanité » sont visibles, comme le drapeau à quatre barres sang et or, le Castillet et le Palais des rois de Majorque à Perpignan. La langue catalane y est présente sur tous les panneaux, indiquant les noms de lieux. Mais curieusement, la présence orale du catalan est beaucoup plus discrète dans l'espace public, et il faut tendre l'oreille sur le marché de la Place Cassagne pour percevoir les consonances catalanes.

Cette enquête a été menée par un observateur non-impliqué, non-locuteur du catalan, jetant un regard relativement neuf sur ces problématiques. L'étude sur les représentations des langues va tenter de repérer comment le conflit diglossique très présent en Espagne trouve ou non un écho auprès de ceux qui ont choisi d'apprendre le Catalan en France.

Les groupes cibles de cette recherche sont constitués par des étudiants. Ils sont eux-mêmes résidents dans le département, et peuvent comme l'enquêteur, être détachés ou impliqués dans la diglossie français-catalan. Leurs représentations des deux langues peuvent-elles être semblables? Existe-t-il une différence d'approche entre ceux qui suivent en licence une option de catalan, et ceux qui se destinent à enseigner dans les écoles bilingues, et qui suivent, à l'intérieur de leur formation initiale d'enseignant du premier degré, un enseignement du catalan, en grande partie en catalan?

On ne saurait, en commençant cette étude, ignorer le creuset linguistique dans lequel s'opèrent ces choix de cursus universitaire, le contexte sociolinguistique n'étant pas étranger aux motivations et aux représentations des étudiants. 


\section{Le contexte sociolinguistique}

\subsection{Des deux côtés des Pyrénées : une histoire et des problématiques différentes}

La Catalogne du nord se rattache à une aire géographique plus vaste s'étendant sur 68000 km², touchant quatre pays européens : la France, l'Espagne, l'Andorre et l'Italie, avec la Sardaigne. La partie française représente donc un peu moins de $6 \%$, de l'espace géographique de rayonnement du catalan. Dans les deux principaux pays, les histoires nationales font que le bilinguisme régional s'exprime de manière différente dans les deux principaux pays. Mais on ne peut pas réellement comprendre les problématiques françaises sans faire référence à celles qui se sont posées pour le voisin espagnol.

La situation espagnole, le grand public l'a appréhendée récemment grâce à l'écho dans les médias de la consultation qui s'est déroulée au mois de novembre 2014, et qui portait sur l'indépendance de la Catalogne. Plus de deux millions de personnes se sont rendues aux urnes, autour de deux questions enchâssées: «Voulez-vous que la Catalogne devienne un État? - En cas de réponse affirmative, voulez-vous que cet État soit indépendant? ». À l'issue du scrutin, non reconnu par le gouvernement espagnol, la réponse était positive à plus de $80 \%$.

Ce référendum informel est un des aboutissements de plusieurs manifestations de masse qui ont ponctué l'année barcelonaise, tous les 11 septembre au cours des trois années précédentes. Celle de 2012, organisée par l'Assemblée Nationale Catalane, a rassemblé près de 1,5 millions de personnes autour du slogan «Catalogne, prochain État de l'Europe». Un an plus tard, c'est deux millions de catalans qui forment une chaîne humaine de 400 kilomètres, métaphore de la voie catalane pour l'indépendance. L'année d'après, 1,8 millions de personnes célèbrent la fête nationale de la Catalogne dans les rues de Barcelone, formant une mosaïque d'un « V » géant de « voter » avec les couleurs du drapeau catalan. 


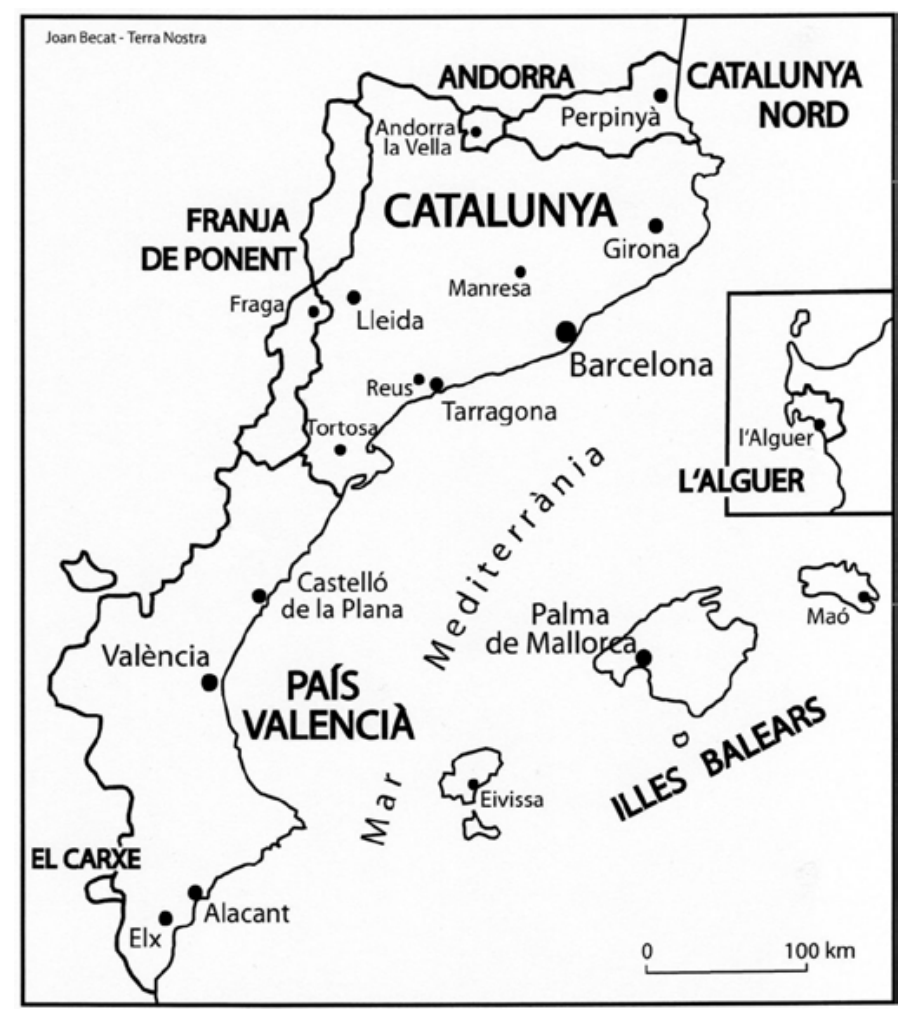

Carte 1 : L'aire linguistique et culturelle catalane ${ }^{1}$

Ces différents points d'orgue récents rappellent l'histoire mouvementée entre la Catalogne et le pouvoir central espagnol, et les conflits qui l'ont jalonnée. La mémoire collective a fait du Moyenâge une sorte d'âge d'or, avec le rêve d'un empire pyrénéo-méditerranéen, la « cataloccitanie », ses symboles forts, le drapeau et la croix occitane. Elle retient aussi de manière négative le XV siècle, lorsque la Catalogne tombe sous l'autorité castillane, davantage préoccupée du nouveau continent à découvrir. C'est le début d'un repli, qui n'empêchera pas la France et l'Espagne de se disputer cette région, conflits aboutissant à la partition lors du Traité des Pyrénées en 1659, traumatisme vécu de la même manière de part et d'autre de la frontière².

Il faut attendre le XIXe siècle pour que la Catalogne connaisse un nouvel essor dû à une industrialisation précoce, qui se manifeste par le « catalanisme ». Les partis indépendantistes font accéder la région au statut d'autonomie en 1932, ressuscitant le terme médiéval de " generalitat»; de manière éphémère puisque la guerre civile y met un terme en 1939. Ce n'est qu'en 1978, deux ans après la fin du régime dictatorial et la mort du Général Franco, que la Généralité de Catalogne est rétablie, avec une importante autonomie, renforcée en 2006. Selon le préambule de son statut, la Catalogne est

1. Carte issue du site http://www.maison-pays-catalans.eu/presentation/la-catalogne-et-les-pays-catalans/. [Page consultée le 11 avril 2015.]

2. Source : http://www.tv5monde.com/cms/chaîne-francophone/info/Les-dossiers-de-la-redaction/Espagne. p29527-Espagne-la-victoire-en-trompe-1-il-de-l-independantisme-catalan.htm. [Page consultée le 22 novembre 2014.] 
reconnue nationalité d'Espagne, terme ambigu qui génère des différences d'interprétation et des contestations sur le plan politique. En juillet 2010, le Tribunal constitutionnel récuse les nouveaux statuts comme non conformes à la constitution sur plusieurs points tels que la notion de nation, de justice autonome ou de fiscalité. C'est le détonateur du conflit actuel ${ }^{3}$.

\subsection{La langue : un enjeu central}

On comprend que la langue catalane, ciment de cet espace politico-géographique, soit un élément majeur dans cette volonté d'indépendance et de singularité face à un État plus fort ou davantage constitué. Son progrès ou son déclin suit le cours des événements historiques et des évolutions politiques. Lors de la "Renaixenca» du milieu du XIXe siècle, le catalan retrouve progressivement son statut de langue officielle. Cette période est marquée par tout un mouvement de défense et de récupération de la langue grâce, notamment à la production littéraire et à l'enseignement. Mais cette renaissance est stoppée par les périodes de dictature, qui feront tout pour faire disparaître le catalan, tout comme le basque ou le galicien. «Sera dénoncée toute personne qui enfreindra l'interdiction de parler toute langue ou dialecte autre que le castillan », est une des mesures du franquisme (Billiez, $2001: 60)$.

L'arsenal des actes répressifs se porte sur la vie politique, l'enseignement et les médias d'où le catalan est banni ; les intellectuels sont poursuivis, les collections scientifiques détruites. Tout est fait pour que les Catalans se castillanisent. Cependant le travail de récupération ${ }^{4}$ entrepris auparavant n'avait pas été effectué en vain puisque la résistance du groupe linguistique catalan, associé à sa force économique, a finalement eu raison de cette situation. Depuis 1979, l'article 3 de la loi de normalisation votée par le parlement catalan stipule que : "La langue de la Catalogne est le catalan. Le catalan est la langue officielle de la Catalogne avec le castillan, langue officielle de l'ensemble de l'État espagnol » (Boyer, 1996 : 126).

\subsection{La Catalogne : un cas d'école pour la sociolinguistique}

Toutes ces menaces existentielles [...] depuis le siècle dernier, provoquent une attitude de solidarité avec le peuple de la part d'un très grand nombre de savants catalans, un engagement dans la pratique de recherche que l'on ne rencontre sans doute que rarement. (Kremnitz, $1980: 22)$

C'est notamment grâce aux travaux des linguistes catalans, notamment Henri Boyer, que le concept de bilinguisme, entendu comme simple présence historique de deux langues, a été remplacé par

\footnotetext{
3. Même référence de site.

4. Notamment l'œuvre de Pompeu Fabra, sous l'impulsion de l'Institut d'études catalanes, qui pose en 1907 les normes du catalan contemporain en publiant les normes orthographiques, la première grammaire normative et le dictionnaire général de la langue catalane.
} 
celui de diglossie, plus dynamique car il porte en lui le caractère évolutif et instable inhérent aux situations de conflit. Une langue dominante se développe au détriment d'une langue dominée, l'issue de ce rapport de forces étant le processus de substitution. La situation catalane offre un bel exemple de cette dynamique.

Il y a de vieilles langues écrites depuis le Moyen Âge qui, après une période de "décadence", regagnent du terrain et semblent se trouver sur le chemin de ce qu'on appelle parfois la normalisation; c'est le cas du catalan. (Kremnitz, 1998:2)

L'école catalane de sociolinguistique a également travaillé sur le concept de normalisation, processus décrit ainsi par Henri Boyer:

Celle-ci [la langue dominée], grâce à une prise de conscience collective et une politique linguistique efficace, reconquiert les territoires qu'elle a dû céder à sa concurrente (dans la vie publique, l'activité politique, l'administration, les médias, le commerce etc.), c'est-à-dire une extension normale des usages sociaux et officiels de sorte qu'elle ne soit plus seulement utilisée pour les échanges privés. (Boyer, 1996: 125)

C'est ce qui s'est passé pour le catalan puisque du point de vue de l'éducation et de l'enseignement, la loi précise: «Tous les enfants de Catalogne, quelle que soit leur langue habituelle au début de leur scolarisation, doivent pouvoir utiliser normalement et correctement le catalan et le castillan à la fin de leurs études primaires $»^{5}$.

Les catalanistes, tout comme les occitanistes, ont aussi travaillé sur le rôle des représentations dans le processus de substitution. La réflexion sur les idéologies linguistiques montre que les langues en présence sont perçues comme des langues inégales de par leur nature et leurs vertus intrinsèques, cette inégalité étant compensée au niveau mythique par des qualités attribuées à la langue dominée, qui jouent en quelque sorte le rôle de compensation consolatrice. La langue dominée est la langue du cœur, alors que la langue dominante est celle de l'esprit. Ces représentations typiques des situations de diglossie favorisent le processus de substitution et pas du tout celui de normalisation.

Comment s'effectue la mutation depuis une situation de diglossie, ou de bilinguisme diglossique à une situation de bilinguisme neutre? Les pratiques langagières passent par « une exacerbation dans l'expression des représentations sociolinguistiques ». En témoigne la signalétique urbaine : «Aussi la rue, les médias, l'école..., sont-ils des lieux où se multiplient les signes d'une inversion de légitimité sur le marché linguistique en Catalogne » (Boyer, 1996: 130). Ces signes sont moins marqués dans les médias, très peu présents dans la presse écrite, assez peu à la radio. En revanche, la télévision dispose de deux chaînes entièrement en catalan ; le livre catalan aussi se porte bien.

5. Article 14, 14e alinéa de la « Llei 7/1983 de 18 d’abril, de Normalizació Lingüística a Catalunya ». 
Du point de vue des usages individuels, on constate, entre 1975 et 1986, une progression des pratiques orales, davantage dans la compréhension que dans la production. En outre, dans la région de Barcelone, le catalan est vu comme la langue de l'intégration et de la réussite sociale. Savoir parler le catalan est considéré comme un avantage par les populations «immigrées », ce terme désignant aussi des Espagnols d'autres régions.

On peut incontestablement observer une évolution de la situation dans le sens d'un arrêt du processus de substitution linguistique hérité du franquisme et un impact de la politique linguistique surtout sur les représentations et les attitudes. [...] Ce que montre à l'évidence l'ensemble des enquêtes, c'est que « le processus de substitution du catalan par le castillan a été freiné », mais que « cela ne signifie pas que le catalan soit sauvé ».

Aujourd'hui, les responsables souhaitent franchir une nouvelle étape dans la normalisation: faire en sorte que le catalan soit la langue prioritaire (et non plus seulement (co)-officielle). (Boyer, $1996: 134)^{6}$

\subsection{La situation sociolinguistique de la Catalogne du nord}

Même si le français est resté une langue inconnue de la majorité de la population pendant plusieurs siècles, l'isolement de la région a longtemps condamné toute tentative de développement du catalan. Au XVIII siècle, « les catalans du nord doivent se faire à leur nouvelle conjoncture d'ultra-midi français, qui petit à petit va essayer de les couper de leurs bases catalanes plus actives : ils se sentent quelque peu loin de tout et de tous mais se débrouillent» (Peytavi Deixona, 2010 : 8). Ni la Révolution française, ni le passage de l'agriculture régionale de l'autarcie à l'économie de marché n'inverseront la tendance. C'est surtout la fin du XIXe siècle et les lois Jules Ferry qui vont, comme pour beaucoup de langues régionales en France, porter le coup de grâce au catalan.

C'est pour la langue catalane le début de grosses difficultés qu'elle n'avait pas eu à affronter jusque-là ou auxquelles elle avait réussi à faire face. Effectivement, les lois de scolarisation de Jules Ferry (1880-1882) compromettent la pérennisation du catalan jusqu’ici la seule langue parlée par l'écrasante majorité de la population. L'école gratuite, obligatoire et laïque ouvre en quelques générations une brèche immense dans les flancs le la « catalophonie » que les régimes précédents n’avaient même pas su inciser. (Peytavi Deixona, 2010 : 10)

6. Citant Tudela (1986). 
Entre 1945 et 1955, on observe un phénomène nouveau : la totalité de la population, classes modestes comprises, adopte le français comme langue familiale. Si jusque-là les gens avaient accepté la répression systématique du catalan par l'école et ses maîtres formés au centralisme - la situation était le même pour toutes les langues minoritaires de l'Hexagone - à partir de ce moment « ils vont assumer eux-mêmes cette persécution au sein de la famille. [...] Le résultat était, pour ce pays, un cas extrême de diglossie : le catalan était relégué aux travaux humbles et au troisième âge. C'était, disait-on une langue pour aller à la vigne » (Verdaguer, 1999: 29).

Aujourd'hui, les Catalans, enclavés dans ce fond de France, "n’ont pas regardé pendant des décennies en direction de ce Sud catalan défini comme "espagnol" et ressenti comme trop "rouge" ou trop “noir" selon les époques » (Peytavi Deixona, 2010 : 12). L'émergence de l'Europe devenue communautaire et la création de la région Languedoc-Roussillon « ont commencé à faire regarder à nouveau au-delà des Albères. [...] La volonté en hausse de transmettre langue et surtout culture [...] des individus qui transmettent leur culture peut-être moins leur langue, souvent envers et contre beaucoup » (Peytavi Deixona, $2010:$ 12).

Et cette culture, les Catalans ont prouvé récemment qu'ils étaient capables de la défendre en s'alliant aux combats de la partie espagnole. «Le processus politique engagé en Catalogne a son influence dans les Pyrénées-Orientales. Le référendum du Sud suscite une motivation nouvelle chez les sympathisants de la Catalogne du Nord », peut-on lire dans le journal La Clau7. En effet, l'ouverture à Perpignan d'un bureau de vote à Perpignan à la Casa de la Generalitat, délégation du gouvernement catalan, pour recevoir les suffrages des quelque 500 catalans de nationalité espagnole est un prétexte à manifestations. La date du 9 novembre est elle-même symbolique puisqu'elle commémore la sinistre partition de la Catalogne, un 7 novembre de l'année 1659.

Dans l'Indépendant ${ }^{8}$ du lundi 10 novembre 2014, on peut lire que sur 500 inscrits, il y a eu 480 votants. "Et que dire des regards attendrissants des militants catalanistes français, présents toute la journée devant les locaux de la Casa, partagés entre la déception de ne pas pouvoir participer au scrutin et la joie de voir leurs homologues, Catalans du Sud, prendre leur destin en mains ». Pour Christophe, «les catalans ont allumé une mèche. Ils veulent enfin être reconnus, comme un peuple à part entière dans l'Europe. On a déjà un hymne, un drapeau, un parlement. II ne nous manque qu'un État».

Par-delà cet engagement culturel et politique, la langue catalane ne fait-elle pas figure de rescapée?

7. Article paru le 7 novembre 2014 dans La Clau, média bilingue diffusé exclusivement sur internet et s'adressant « aux lecteurs sensibles aux modalités modernes de la question régionale et aux enjeux européens ». Source : http://www. la-clau.net/info/9646/les-evenements-de-catalogne-agitent-perpignan-9646. [Page consultée le 22 février 2015.]

8. Principal quotidien régional tiré à plus de 60000 exemplaires. 


\subsection{La politique des langues en Catalogne du Nord}

Considérée depuis la loi Deixonne de 1951 comme une langue pouvant être enseignée à l'école, au même titre que le basque, l'occitan, l'alsacien, le catalan est reconnu depuis 2008 comme langue régionale appartenant au patrimoine de la France, sans pour autant bénéficier d'aucun statut officiel. Cependant, la Région Languedoc-Roussillon a créé un organisme officiel ayant pour mission la promotion de la langue et culture catalane et occitane. Il existe également un Intergroupe Langues et Cultures Régionales au sein du Conseil Régional.

Sur le plan local, la municipalité de Perpignan a promu la langue et la culture catalanes au moyen de différentes actions, comme la création en 1978 du Centre de Documentació i Animació de la Cultura Catalana (CEDACC), qui se compose d'une bibliothèque, d'un service de documentation et d'une salle d'expositions. Le CEDACC a récemment augmenté ses activités suite à la création d'un service de catalan et de consultation linguistique, l'organisation de cours de catalan pour les fonctionnaires municipaux et de cours d'adultes, ainsi que des cours de catalan au niveau préscolaire et dans les écoles primaires (1 000 élèves en 1993-1994). Depuis 1981 il décerne des prix annuels de littérature et audiovisuels ${ }^{9}$.

Les données fournies par une enquête récente ${ }^{10}$ font état que 49 \% des personnes interrogées (environ 140000 personnes) déclarent savoir parler le catalan, bien que 16 \% de celles-ci disent éprouver des difficultés dans l'expression à cause d'un manque de maîtrise. La compétence linguistique augmente de pair avec l'âge : $16 \%$ des $18-24$ ans parlent bien le catalan, contre $73 \%$ chez les plus de 65 ans. Dans les communes de moins de 1000 habitants, 70 \% des gens savent parler le catalan, alors qu'ils ne sont que $39 \%$ à Perpignan. L'usage du catalan est beaucoup plus répandu parmi les agriculteurs (72\%) et les retraités (63\%) que dans les autres secteurs sociaux.

En ce qui concerne l'usage, $66 \%$ des catalanophones déclarent parler le catalan souvent, $23 \%$ occasionnellement, $7 \%$ rarement et $3 \%$ ne le parlent jamais. L'usage du catalan est plus fréquent au sein de la famille ou avec les amis (66 \%) qu'avec les personnes âgées (37\%) ou lors des échanges courants (37\%). En outre, $61 \%$ de catalanophones considèrent que leur pratique langagière est stable ; $21 \%$ pensent qu'elle régresse et $19 \%$ pensent qu'elle augmente. À l'exception des 18-24 ans, plus on est jeune, et plus on pense que la pratique augmente. Quelque 55000 personnes déclarent savoir lire le catalan sans difficulté, tandis que les agriculteurs et les étudiants sont les plus nombreux à savoir l'écrire (25\%).

9. Données recueillies sur le site http://www.uoc.es/euromosaic/web/document/catala/fr/i5/i5.html. [Page consultée le 16 mai 2014.]

10. Données recueillies sur le site mentionné ci-dessus, faisant probablement référence à l'enquête de l'INSEE de 2002 «Langues régionales, langues étrangères: de l'héritage à la pratique ». 
Les collectivités territoriales entretiennent des liens avec la Generalitat de Catalunya par le biais d'accords transfrontaliers : le Conseil municipal de Perpignan, pour la promotion et la diffusion de la langue catalane; le Conseil général des Pyrénées-Orientales et l'Université de Perpignan pour les aspects linguistiques; la Chambre de commerce et d'industrie pour la réalisation des épreuves du certificat international de catalan. À l'inverse, la Generalitat collabore avec les organismes français pour l'élaboration de cours de catalan, l'échange de professeurs, la recherche sociolinguistique et l'élaboration de matériels didactiques, etc. Il existe aussi une convention entre les petites universités de tous les pays de langue catalane, dont celle de Perpignan, afin de décerner des diplômes communs de $3^{\mathrm{e}}$ cycle ${ }^{11}$.

\subsection{Le catalan au niveau de l'enseignement universitaire}

L'Institut franco-catalan transfrontalier (IFCT), situé à la maison du pays catalan, Casa dels Països Catalans, compte six enseignants titulaires et 316 étudiants en 2013-2014 ${ }^{12}$. Cette composante de l'Université Via Domitia de Perpignan (UPVD) organise les enseignements et les diplômes nationaux et des diplômes d'université relatifs aux études catalanes et aux questions transfrontalières licences, licence professionnelle, master, master professionnel, master MEEF, doctorat, certificats de langue catalane.

L'IFCT réunit un Département d'Études Catalanes, chargé des enseignements de licence (licence de catalan et licence professionnelle de traduction-interprétation) et des diplômes d'université, un Service de télé-enseignement de catalan, le SETELCAT, ainsi qu'un Laboratoire habilité, l'ICRESS, Institut catalan de recherche en sciences sociales (Équipe d'Accueil n 3681 ), responsable de la mention de master Études européennes et internationales : master recherche Études catalanes et master professionnel de Relations transfrontalières.

L'IFTC bénéficie non seulement des Institutions et collectivités territoriales françaises comme n'importe quel organisme, mais aussi d'entités catalanes ou transfrontalières dont certaines ont un siège au conseil de l'IFTC. Citons entre autres l'Institut d'Estudis Catalans (Académie de la langue catalane), dont le siège est à Barcelone et qui travailla à doter la langue de structures stables et de normes orthographiques. Citons encore l'ALPEC (Association pour l'enseignement du catalan), qui a vocation de réunir les enseignants et parents d'élèves des deux pays qui soutiennent l'enseignement bilingue public ou privé.

11. «Le catalan en France », http://www.uoc.es/euromosaic.html. [Page consultée le 16 mai 2014.]

12. Source : https://www.univ-perp.fr/fr/menu/l-upvd/les-facultes-instituts-et-centres/institut-franco-catalan-transfrontalier-ifct-/institut-franco-catala-transfronterer-institut-franco-catalan-transfrontalier-4616.kjsp. [Page consultée le 22 février 2015.] 
La formation des enseignants bilingues s'effectue dans le cadre d'un Master Métiers de l'éducation, de l'enseignement et de la formation, au sein de l'ESPE Languedoc-Roussillon. À Perpignan, deux unités de formation se partagent la formation des futurs professeurs du premier et du second degré, l'une à l'UPVD, l'autre à la Faculté d'éducation qui dépend de l'Université de Montpellier.

En ce qui concerne la formation des adultes et la formation permanente, des initiatives ont été mises en place par le GREC, l'Associació Politècnica, l'Escola Catalana, le Centre Cultural Català et le Centre de Documentació i d'Animació de la Cultura Catalana (CEDACC) de la Ville de Perpignan; la filiale d'Òmnium Cultural (Òmnium Cultural Catalunya-Nord); et le Servei de Llengua de l'Institut Franco-Català (IFC) de l'Université de Perpignan.

\section{Méthodologie contextualisée de l'enquête}

\subsection{L'entretien exploratoire : une étape décisive}

Un entretien exploratoire avec un groupe témoin s'est déroulé à la fin de l'année universitaire précédant le début de l'enquête, au mois de juin, auprès d'étudiants de Master 2, donc en fin de leur parcours de formation, et de ce fait, ils n'étaient pas susceptibles de faire partie de l'échantillon d'enquêtés. Il a eu lieu au début d'un cours, alors qu'ils n'étaient pas prévenus de cette initiative. Sans doute est-il nécessaire de s'y prendre autrement et de bien expliciter la démarche afin de s'assurer de l'assentiment des membres du groupe. Cet entretien m'est apparu assez décevant par son côté très artificiel et très loin de la conversation à bâtons rompus, formule la plus souhaitable. Les étudiants sont restés sur une certaine réserve, se contentant de réponses stéréotypées, d'autant plus que la stratégie de guidage « Pour moi le catalan c'est la langue de... c'est la langue des... c'est une langue qui... » est apparue comme peu favorable à des échanges naturels. J'ai donc doublé cet entretien d'un second, toujours avec des étudiants de catalan, mais sans guidage. 
Il a résulté de ces entretiens somme toute très complémentaires, la formulation de quinze items, reprenant sans les trahir le teneur des deux entretiens:

\section{CATALAN : 15 items}

1. Une langue menacée, à défendre.

2. Une langue qui ouvre sur l'Europe.

3. Récemment le catalan a profité des réseaux sociaux.

4. C'est une langue romane.

5. Il n’y a pas de loi qui protège le catalan.

6. C'est une langue à part entière, pas un dialecte.

7. C'est une langue parlée surtout en Espagne.

8. Le catalan s'enracine dans la culture familiale.

9. C'est une langue facile à apprendre à l'école.

10. Le catalan retrouve un certain souffle.

11. C'est une langue territoriale liée à une culture.

12. Tout le monde se comprend malgré les dialectes..

13. Le catalan est davantage reconnu en Espagne qu'en France.

14. Sans le Catalan, on ne peut pas connaître l'histoire ou la sociologie de la Catalogne.

15. C'est une langue très riche car elle a beaucoup de dialectes.

\section{FRANÇAIS : 15 items}

1. Une langue européenne et internationale.

2. La langue qui a la grammaire la plus horrible du monde.

3. Une langue pratiquée quotidiennement.

4. Une langue bien maîtrisée.

5. Le français, c'est la langue de l'amour.

6. Une langue historique, chargée de culture.

7. La langue des Jeux Olympiques.

8. La langue responsable de la disparition du catalan.

9. C'est la langue des apprentissages. 
10. Le français empêche l'apprentissage d'une autre langue.

11. Le français permet d'apprendre la grande littérature.

12. Tout le commerce, tout le tourisme est en français.

13. C'est une langue nécessaire si on veut accéder à la culture.

14. La littérature française exerce un poids sur le Catalan.

15. C'est une base pour traduire en catalan les littératures étrangères.

Dans ces formulations, il y a des affirmations qui sont plus opérationnelles que d'autres dans le cadre d'une étude sur les représentations. Des items tels que «C'est une langue romane » ou bien « c'est une langue parlée en Espagne » sont essentiellement de l'ordre de la définition ou de la constatation et elles ne contiennent pas en elles-mêmes un avis qui puisse être discuté. De même la formule « une langue pratiquée quotidiennement » relève davantage des pratiques individuelles et n’est pas révélatrice de représentations sur la langue. Cependant, il n'est pas apparu nécessaire de supprimer ou remplacer ces items.

\subsection{Quel public?}

Lorsqu'on exerce en milieu universitaire, les étudiants sont un public de choix, facilement accessible puisqu'il suffit de mobiliser un petit moment, une vingtaine de minutes au début ou à la fin d'un cours. Si ceux de la Faculté d'Éducation font partie de mon environnement proche, il a fallu le concours d'un collègue de l'Université de Perpignan pour toucher ceux qui sont inscrits dans un cursus comprenant, à des titres divers, un enseignement de la langue régionale. Voici les différents sous-groupes qui composent notre corpus:

(1) Les étudiants de la faculté d'éducation (FdE), inscrits dans un cursus bilingue, qui reçoivent un enseignement spécifique de la langue catalane, mais aussi en langue catalane ainsi que des enseignements sur la didactique des langues et spécialement du catalan. Ils suivent par ailleurs tous les autres enseignements propres au Master «Métiers de l'éducation, de l'enseignement et de la formation (MEEF). Ces étudiants sont presque tous bilingues, au tout au moins ont une connaissance de base du catalan, à défaut d'une véritable pratique. Lors des cours, la langue de communication est, le plus souvent, le catalan mais sans exclusive.

(2) Les étudiants de l'Université de Perpignan (UPVD) ont été répartis en deux catégories : ceux qui, au sein de l'« Institut franco-catalan transfrontalier » suivent la licence et le Master de Catalan; et ceux qui, à l'intérieur de leur filière, suivent sous forme de langue étrangère ou comme option, un enseignement de la langue catalane. Ceux-ci viennent de tous horizons, y compris de l'étranger, ne sont en général pas catalanophones et leurs motivations peuvent être très diverses. Qui sont ces étudiants non-spécialistes? 
- Les non-spécialistes 1 ère année : ce sont en très grande majorité des femmes (82 \%) âgées en moyenne de moins de vingt ans, donc de jeunes étudiantes. Elles sont $40 \%$ à déclarer l'anglais et l'espagnol comme première et seconde langue, $24 \%$ à déclarer espagnol et catalan parmi leurs deux premières langues ; la même proportion est locuteur du catalan. Mais il ne s'agit pas là comme cela est pensé initialement à la $L 1$ = langue " maternelle », mais de la première langue étrangère étudiée (ambiguïté de la proposition). Elles suivent des formations principalement en lettres (langues étrangères appliquées - LEA), le catalan constitue leur troisième langue étrangère, sous forme d'option, les autres options étant l'allemand ou le portugais.

- Les non-spécialistes de $2^{\text {ème }}$ année : C'est un public assez semblable, composé en majorité de jeunes étudiantes de vingt ans. Mais les hommes sont un peu plus nombreux en proportion, et ce groupe accueille quatre étudiants plus âgés : 54, 61, 63 et 67 ans. Le répertoire verbal majoritaire est $L 1$ : anglais, $L 2$ : espagnol (même réserve que pour le groupe précédent). Les locuteurs du catalan sont au nombre de cinq. La moitié suit des études de langues étrangères appliquées, le catalan est une option. Une donnée demeure dans l'ambigüité : ceux qui déclarent le catalan en L1 ou L2 sont-ils locuteurs habituel de cette langue, ou la mentionnent-ils parce qu'elle fait partie de leur cursus universitaire en première ou seconde langue étudiée?

(3) Le dernier groupe à avoir été interrogé n'est pas constitué d'étudiants mais d'adultes concernés par la pratique ou l'apprentissage du catalan. Le questionnaire a été transmis par une étudiante de la FdE aux parents d'élèves d'une école bilingue de Perpignan, alors qu'elle s'y trouvait en stage. Douze personnes ont répondu, onze d'entre elles sont des femmes, l'âge moyen de cet échantillon est de 48 ans. Tous sont francophones, même si trois ne déclarent pas le français comme langue « usuelle » mais le catalan, et deux mentionnent leur bilinguisme français/catalan. Huit de ces personnes déclarent pratiquer le catalan, et cinq d'entre elles sont aussi locuteurs de l'espagnol. Cependant, trois d'entre elles seulement se déclarent explicitement apprenant de catalan, les autres n'ayant pas renseigné cette rubrique.

\subsection{Méthodologie du questionnaire}

Les étudiants de la FDE ont rempli le questionnaire en ma présence. Pour l'UPVD, j'ai reçu le concours de M. Baylac qui a diffusé le questionnaire auprès de ses étudiants et a pris le soin d'en expliciter la démarche, contrôler la passation et collecter les réponses au moment de chacun de ses cours ${ }^{13}$. Les questionnaires ont été complétés avec beaucoup de sérieux, et en respectant les consignes de notation. Quelques réponses seulement ont été écartées car la procédure n’avait pas été comprise.

13. Je remercie chaleureusement M. Ala Baylac d'avoir pris de son temps pour que l'enquête soit largement diffusée. Je remercie aussi ma collègue de la FdE professeur de catalan, Mme Rita Peix pour ses conseils bibliographiques et les discussions informelles sur le sujet. 
En tout, 174 personnes ont répondu à l'enquête : 32 étudiants de filière catalane, 15 de la faculté d'éducation se préparant au métier d'enseignant au sein du Master MEEF, filière bilingue ; 24 de I'UPVD suivant une licence de catalan : 9 en L1, 10 en L2 et 5 en L3. 55 étudiants «non-spécialistes » de lère année, inscrits à l'UPVD en option catalan. 29 étudiants « non-spécialistes » de Zème année, inscrits à l'UPVD en option catalan et 12 parents d'élèves.

Comment saisir les résultats? Il est apparu préférable de distinguer d'une part les étudiants suivant un cursus universitaire bilingue, en catalan et français, avec des enseignements en catalan, en principe catalophones en langue première ou seconde, des autres étudiants ayant ajouté le catalan dans leurs matières enseignées. En effet leur position par rapport à la langue, ainsi que leurs représentations devraient être différentes et on les imagine dans une position plus impliquée, voire militante par rapport à une langue qui leur appartient et qui demeure minorée. Les étudiants de la FdE ont été dans un premier temps intégrés au corpus des bilingues; l'année suivante, l'enquête concernant 15 étudiants de master 1 a fait l'objet d'un traitement à part.

Cinq saisies ont donc été effectuées, la première concernant les étudiants catalophones, qu'ils soient de la FdE ou de l'UPVD, la seconde les étudiants UPVD « non spécialistes » suivant une option en catalan en $\mathrm{M} 1$, la troisième les étudiants de même catégorie suivant un Master $2^{e}$ année ; la quatrième concernait les étudiants de la FdE et la cinquième le groupe des parents d'élèves.

\section{Résultats et analyse de l'enquête}

Cette analyse s'effectuera en deux temps. Une première analyse sera réalisée à partir de la comparaison entre les résultats de deux groupes seulement, sur cinq. Il s'agit:

- des étudiants des filières catalanes (UPVD et FdE confondus), année 2013-2014,

- des étudiants «non-spécialistes » de l'UPVD, année 2013-2014.

Cette comparaison est en elle-même déjà assez significative. Elle sera affinée par l'analyse des graphiques des étudiants de la FdE, puis des apprenants adultes du catalan.

Bien que la même enquête ait été menée conjointement avec les mêmes publics au sujet du français, ce sont principalement les représentations du catalan qui seront le sujet central de cette analyse. 


\subsection{L'enquête sur le catalan}

Observation des deux graphes des étudiants de l’UPVD (non-spécialistes et filières) :

Ce qui apparaît manifeste sur le graphe des étudiants catalophones, c'est l'item 6 « Une langue à part entière, pas un dialecte », qui apparaît sur le tableau sous sa forme résumée "véritable langue ». Le couple consensus/adhésion est fort $(1,47 ; 0,089)$, ce qui montre que beaucoup de ces étudiants ont choisi cette affirmation comme très représentative pour le catalan. La reconnaissance du statut de leur langue semble donc très importante.

Dans ce qui apparaît comme un trio de tête, on trouve l'affirmation 11 «C'est une langue territoriale, liée à une culture », apparaissant sur le graphe sous la forme abrégée « Terroir, culture », ainsi que l'item 1 « Une langue menacée, à défendre ». Il convient d'inclure cette dernière, bien qu'elle ne fasse pas partie de la couronne centrale $(0,91 ; 0,078)$, parce qu'elle est bien détachée de la position des autres items.

Très différent apparaît le graphe des étudiants UPVD non-spécialistes de Master 1. D’abord il n’apparaît pas d'adhésion très marquée. Un groupe de six items ne dépasse pas le score 1,00. On y retrouve, à peu près à la même place l'affirmation 11 «langue territoriale... », indice d'adhésion égal à 1,00, mais les deux autres affirmations, 6 et 1, sont à une place bien différente, 0,49 pour la première et 0,13 pour la seconde. Le fait que le catalan soit une véritable langue, et qu'elle puisse être menacée ne remportent qu'une adhésion faible et ne font guère consensus. On remarque chez les étudiants non-spécialistes de M2 une différence notable dans les indices d'adhésion de ces deux mêmes items 6 et 1 (respectivement 0,9 et 0,31), ce qui tendrait à montrer qu'au fur et à mesure de son apprentissage le rapport à la langue évolue dans le sens d'une meilleure conscience de son statut, ainsi que de la nécessité d’agir pour sa défense et sa préservation.

Au sujet de l'item 11, «Une langue territoriale, liée à une culture », il se peut que cette proposition, dans la formulation adoptée ait été comprise différemment suivant les groupes, les uns étant sensibles à l'aspect culturel, donc une certaine forme de rayonnement, alors que les autres auraient adhéré au fait que le catalan soit très circonscrit à un terroir, une aire géographique qui limite son usage. En tous cas cette proposition peut être mise en opposition avec les deux items qui traduisent un rayonnement du catalan, une extension hors des frontières géographiques que sont les propositions suivantes « une langue qui ouvre sur l'Europe » et « récemment cette langue a profité des réseaux sociaux ». Ces items obtiennent des notes négatives de la part des deux groupes étudiants non-spécialistes de M1 et M2 confondus.

Plusieurs items représentent un consensus négatif de la part de l'ensemble des groupes. Il s'agit de l'item 10 « une langue qui retrouve un certain souffle », l'item 5 « II n'y a pas de loi qui protège le catalan » et l'item 14 «Sans le Catalan, on ne peut pas connaitre l'histoire ou la sociologie de la Catalogne ». L'item 5 peut être rapproché d'une manière paradoxale avec l'item 1 « langue me- 
nacée ». Le fait que la langue soit perçue comme menacée n'indique pas qu'il n'existe pas de lois pour la protéger. Tout au moins la question de la protection n'apparaît pas comme une affirmation qui recueille l'adhésion. Pour ce qui est de l'item 14, la formulation peut ne pas apparaitre comme pertinente, même pour un catalophone, sans doute parce que l'histoire ou la sociologie de la région ne passe pas exclusivement par la connaissance de la langue.

D’autres affirmations se situent dans la zone médiane, ni forte adhésion, ni fort consensus. Il s'agit par exemple de l'item 3, «Récemment le catalan a profité des réseaux sociaux ». Cette proposition émanant du groupe témoin des étudiants de la FdE n'a pas été valorisée ni par les catalophones, ni par les non-spécialistes. II en est de même pour l'item 12 « Tout le monde se comprend malgré les dialectes », ainsi que l'item 15, «C'est une langue riche qui a beaucoup de dialectes », malgré un rejet un peu moins faible pour cette affirmation de la part des catalophones. La facilité d'apprentissage «Une langue facile à apprendre à l'école » (item 9), se situe dans les valeurs négatives sauf pour les étudiants non-spécialistes de seconde année, mais avec un score d'adhésion faible. Quant à l'affirmation 7, «c'est une langue parlée surtout en Espagne », elle est davantage de l'ordre de la constatation que de la représentation. Cependant le score est franchement négatif pour les catalophones et très positif pour les étudiants non-spécialistes. Simple affirmation pour ces derniers, peut-être est-elle davantage source de friction pour les premiers en raison du clivage régional Catalogne/Espagne qui cristallise les rivalités de langue.

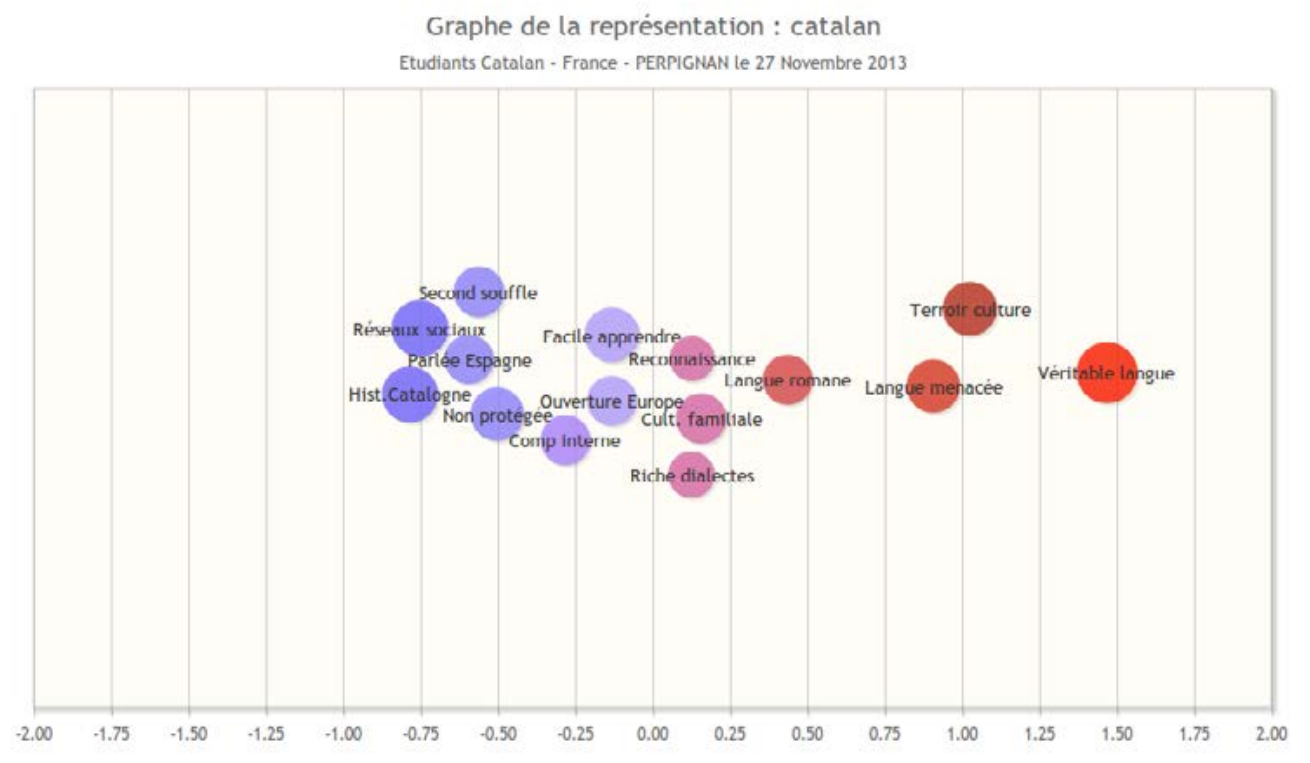

Graphe 1 : Représentation du catalan chez les étudiants des filières catalanes 


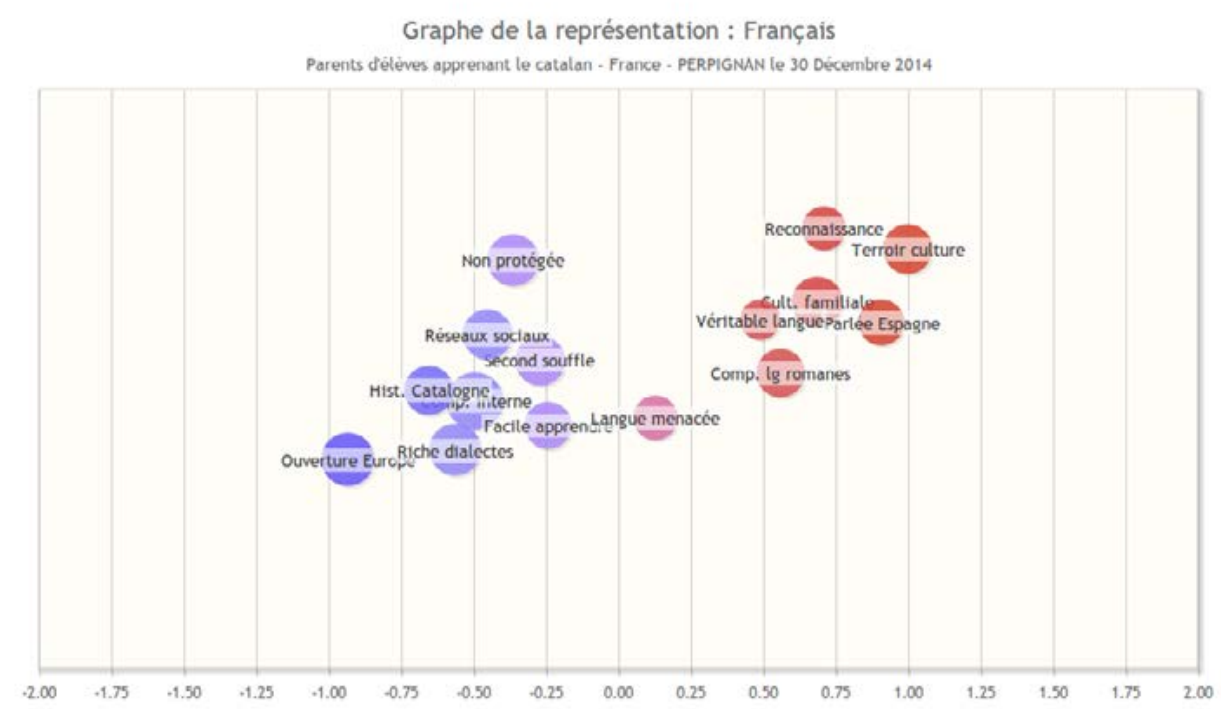

Graphe 2 : Représentation du catalan chez les étudiants non spécialistes

L'observation des résultats des enquêtes menées auprès des autres groupes permettent s'affiner cette analyse. Le graphe des étudiants non spécialistes de la deuxième année de Master diffère peu de celui des étudiants de Master 1 . En revanche, celui des étudiants de l'IUFM ressemble fort à celui des étudiants des filières catalan ; les deux sont comparables à celui des parents d'élèves.

L'observation du seul item 6, « Une langue à part entière, pas un dialecte », est assez révélatrice. En effet, non seulement le couple adhésion/consensus diffère d'un sous groupe à l'autre, mais de plus il semble évoluer en fonction d'un critère qu'on pourrait appeler le « degré d'implication dans les études du catalan ». On peut repérer cette évolution à l'aide du tableau suivant:

Tableau 1 : Résultats pour l'item 6

\begin{tabular}{|c|c|c|}
\hline Item $\mathbf{6}$ « Une langue à part entière, pas un dialecte » & $\begin{array}{c}\text { Score } \\
\text { d'adhésion }\end{array}$ & Score de consensus \\
\hline 1 - UPVD - Étudiants non-spécialistes - 1 $^{\text {ère }}$ année & 0,49 & 0,062 \\
\hline 2 - UPVD - Étudiants non-spécialistes - 2 ${ }^{\text {ème }}$ année & 0,9 & 0,072 \\
\hline 3 - Faculté d'Éducation - Master 1 - MEEF & 1,29 & 0,082 \\
\hline 4 - UPVD - Filières catalanes & 1,47 & 0,089 \\
\hline 5 - Parents d'élèves et apprenants catalan ${ }^{\star}$ & 1,83 & 0,187 \\
\hline
\end{tabular}

* Le petit nombre d'enquêtés rend les résultats moins significatifs. 
En ce qui concerne les deux autres items remarqués dans la couronne centrale pour les étudiants catalanistes, ils suivent une évolution similaire. Cela est vrai surtout pour l'item 1, « Une langue menacée, à défendre », un peu moins pour l'item 11, «C'est une langue territoriale, liée à une culture »:

- Item $1.1:(0,13 ; 0,069)-2:(0,31 ; 0,069)-3:(0,47 ; 0,069)-4:(0,91 ; 0,078)-5:(0,58 ; 0,072)$

- Item $11.1:(1,00 ; 0,077)-2:(0,93 ; 0,07)-3:(0,88 ; 0,066)-4:(1,03 ; 0,079)-5:(0,67 ; 0,076)$

Pour ce qui est des items recueillant les scores adhésion/consensus les plus bas, visualisables sur les graphes par la couleur bleue, les différents groupes sont à peu près d'accord pour choisir comme propositions « qui paraissent le moins bien évoquer le catalan », les affirmations suivantes, ici évoquées de manière abrégée : 2 , ouverture vers l'Europe ; 3 , réseaux sociaux; 5 , non protégée ; 9 , facilité d'apprentissage; 10 , second souffle; 12 , intercompréhension interne; 13 , reconnaissance; 14 , histoire de la Catalogne; 15 , riche de ses dialectes.

\subsection{Les résultats de l'enquête sur le français (tous les sous-groupes)}

Les réponses sont davantage homogènes entre les groupes, que pour le catalan. Pour chacun on trouve dans les items de tête, témoignant d'un bon score d'adhésion et de consensus les trois affirmations suivantes:

- une langue européenne et internationale (item 1)

- la langue pratiquée quotidiennement (item 3)

- une langue historique, chargée de culture (item 6).

Ensuite, viennent les affirmations liées à la difficulté de la langue, ce sont les items 2, «La langue qui a la grammaire la plus horrible du monde », 'item 4 « Une langue bien maîtrisée », relevant plutôt des compétences individuelles que des représentations, et l'item 11 « Le français permet d'apprendre la grande littérature ». Ce sont des caractéristiques généralement attribuées à une langue majoritaire ou dominante, et elles expriment des représentations souvent attribuées à la langue française.

On trouve également une grande homogénéité en ce qui concerne les affirmations qui recueillent le moins d'adhésion. Le critère d'exclusion, exprimé par l'affirmation « Le français empêche l'apprentissage d'une autre langue » (item 10) est massivement rejeté, du moins dans cette formulation. II obtient même un fort consensus négatif de la part des étudiants non-spécialistes de $2^{e}$ année. Cela semble contredire le fait qu'on attribue souvent à la langue française et à sa complexité, les difficultés éprouvées par beaucoup de français d'accéder à l'apprentissage d'une langue étrangère. De même la proposition de la pression qu'exercerait le français sur le catalan en matière de littérature "La littérature française exerce un poids sur le Catalan », (item 14) n'obtient pas d'adhésion. Dans le même ordre d'idée, l'expression « langue responsable de la disparition du catalan » (item 8) est unanimement rejetée, et en tout cas se situe dans les choix négatifs. Enfin dans les items qui remportent 
très peu l'adhésion des trois groupes on trouve les affirmations suivantes: « la langue de l'amour » (item 5), « la langue des Jeux Olympiques » (item 7) et « Tout le commerce, tout le tourisme est en français » (item 12), ainsi que «c'est une base pour traduire les littératures étrangères » (item 15).

En ce qui concerne le français, on ne remarque pas de différence particulière entre les représentations des locuteurs catalans et les étudiants non-spécialistes. Pas de changements notables pour ce qui est des autres sous-groupes.

Ces résultats posent question si on essaie d'établir des liens entre les enquêtes sur le français et celles du catalan, menées auprès, rappelons-le, des mêmes personnes. Si les choix formulés à propos du catalan divergent, notamment en fonction du degré d'implication ou familiarité ou connaissance de la langue, celles concernant le français sont assez semblables. Pour les catalophones, la question de la reconnaissance d'une véritable langue dans une situation précaire semble importante, mais si la langue semble menacée et ce n'est en apparence pas par la langue française, ce que contredisent les faits historiques. Le français semble ne pas exercer de pression, ni par le rayonnement de sa littérature, ni par sa présence comme langue de scolarisation, ni par la complexité de son apprentissage.

\section{Conclusion}

À l'issue de cette première enquête sur les représentations des apprenants français de catalan quelques conclusions peuvent être annoncées. Plus on est familier de la langue, locuteur natif, résident dans la région, impliqué dans une démarche professionnelle de futur enseignant, étudiant des filières catalanes, parents d'élèves des écoles bilingues ou apprenant adulte sur motivation personnelle, plus le statut positif et valorisé de la langue catalane va s'exprimer.

Tout apprenant quel qu'il soit a conscience de la précarité de la langue et de la nécessité de la défendre. Mais contre quelle menace, contre quel adversaire? Est-ce la langue française ? Cela ne ressort pas des résultats de cette enquête puisque les affirmations qui décrivent l'aspect glottophagique du français sont peu prises en compte : elles sont plutôt rejetées, mais avec un consensus faible. Serait-ce alors le castillan? L'histoire de la diglossie montre à quel point le conflit a été lourd d'implications politiques, et à quel point dans l'Espagne actuelle, la langue est au cœur des mouvements de revendication identitaires et indépendantistes. On sait aussi la solidarité qui existe entre la catalogne et sa partie septentrionale; on sait enfin la conscience de tout locuteur du catalan éprouve envers l'appartenance à un espace géographique et culturel vaste, ce qui se traduit d'ailleurs par la place consensuelle de l'affirmation qui ancre le catalan dans un territoire et l'enracine dans une culture, partagée.

Ce sont donc bien les termes d'un conflit diglossique qui s'expriment ici, mais de manière atténuée. Il apparaît comme le témoin ou l'écho d'un conflit à la fois très proche et plus sévère. Le processus de normalisation, bien qu'ayant suivi des étapes comparables dans le temps, avec la situation espagnole, se pose pourtant en termes différents. Si l'on peut parler du catalan comme d'une langue res- 
capée on constate que sa renaissance passe assez peu par un usage oral dans les pratiques sociales, notamment dans l'espace public. Elle est surtout le fait d'une participation aux symboles culturels régionaux, dont la transmission patrimoniale est très forte. Ceux qui apparaissent motivés par la défense de cette langue sont en définitive ceux qui la redécouvrent, et qui parfois militent au point de vouloir l'enseigner, la transmettre. Néanmoins, la plupart des locuteurs de cette grande langue transfrontalière conservent le sentiment d'appartenir à un espace que la barrière des Pyrénées ne saurait fractionner. 


\section{Références}

Billiez, Jacqueline (2001), Sociologie du langage, notes de cours, Grenoble, Université Stendhal - Grenoble 3, p. 58-69.

Boyer, Henri (1986), « Diglossie : un concept à l'épreuve du terrain », Lengas, n² 20, p. 21-54.

Boyer, Henri (1987), « Sociolinguistique et politique linguistique, l'exemple catalan », Études de Linguistique Appliquée, nº 65, p. 68-88.

Kremnitz, Georg (1980), « Démarches et particularités de la sociolinguistique catalane », dans Bernard Gardin et Jean Baptiste Marcellesi (dir.), Sociolinguistique : approches, théorie, pratiques. Actes du colloque de Rouen, tome 1, Paris, Presses universitaires de France, p. 21-23.

Kremnitz, Georg (1998), «La recherche (socio)linguistique en domaine occitan », Bulletin de l'Association Internationale d'Études Occitanes, nos 2-3, p. 21-54.

Maurer, Bruno (2013), Représentations sociales des langues en situation multilingue : la méthode combinée, nouvel outil d'enquête, Paris, Éditions des archives contemporaines.

Peytavi Deixona, Joan (2010), « Une histoire de la Catalogne du nord : réflexion sur le parcours millénaire de la catalanité », Collectiu OLIBA : connaitre et comprendre la Catalogne, disponible sur http://oliba.catnord.cat/?page_id=43. [Page consultée le 11 novembre 2014.]

Tudela, Joan (1986), El futur del catalá : una radiografia sociolingüística, Barcelona, Publicacions El Mon.

Verdaguer, Pere (1999), Grammaire de la langue catalane : les origines de la langue, Barcelone, Curial. 